\title{
A Data-Driven Method to Characterize Turbulence- Caused Uncertainty in Wind Power Generation
}

\author{
Jie Zhang*,1, Rishabh Jain ${ }^{2}$, Bri-Mathias Hodge ${ }^{3}$ \\ ${ }^{1}$ University of Texas at Dallas, Richardson, TX 75080, USA \\ ${ }^{2}$ North Carolina State University, Raleigh, NC 27695, USA \\ ${ }^{3}$ National Renewable Energy Laboratory, Golden, CO 80401, USA
}

\begin{abstract}
A data-driven methodology has been developed to analyze how ambient and wake turbulence affect the power generation of wind turbines. Using supervisory control and data acquisition (SCADA) data from a wind plant, we select two sets of wind velocity and power data for turbines on the edge of the plant that resemble (i) an out-of-wake scenario and (ii) an in-wake scenario. For each set of data, two surrogate models are developed to represent the turbine(s) power generation as a function of (i) the wind speed and (ii) the wind speed and turbulence intensity. Three types of uncertainties in turbine power generation are investigated: (i) the uncertainty in power generation with respect to the reported power curve; (ii) the uncertainty in power generation with respect to the estimated power response that accounts for only mean wind speed; and (iii) the uncertainty in power generation with respect to the estimated power response that accounts for both mean wind speed and turbulence intensity. Results show that (i) the turbine(s) generally produces more power under the in-wake scenario than under the out-of-wake scenario; and (ii) there is relatively more uncertainty in the power generation under the in-wake scenario than under the out-of-wake scenario.
\end{abstract}

Keywords: data-driven; surrogate modeling; uncertainty quantification; turbulence intensity; wind distribution

\section{Introduction}

Renewable energy resources have become a primary focus in government policies, in academic research, and in the power industry. Among the renewable energy technologies, wind energy has taken a lead; it currently contributes approximately $4 \%$ of worldwide electricity consumption [1]. Wind energy comes from wind power plants that consist of multiple wind turbines distributed throughout a substantial stretch of land (onshore) or water (offshore). The power generated by a wind plant is an intricate function of the configuration and location of the individual wind turbines. The flow pattern inside a wind plant is complex, primarily due to the wake effects and the highly turbulent flow. Wake losses lead to significant energy loss, especially in large-scale wind plants. The average wake loss is approximately 5\%-20\% of the power output from the whole wind plant, depending on turbine placement and site climatology [2]. The offshore average ambient turbulence is typically between $6 \%$ and $8 \%$ at heights of approximately $50 \mathrm{~m}$; the onshore average is between $10 \%$ and $12 \%$ [3]. Within a wind plant, turbulence is characterized by ambient and wake turbulence. Ambient turbulence is defined as the normal turbulence at the site that would be experienced by a single, stand-alone turbine. Wake turbulence is caused by upwind turbines shading those downstream [4]. In the past years, a number of experimental and computational studies have been performed to investigate different wake characteristics within a wind plant, such as velocity deficit, turbulence intensity, multiple wake interactions, and the wake width and trajectory at various distances downwind [3-14].

In the presence of turbulence, with the same wind speed condition, the same turbine might generate different power under ambient turbulence (when the turbine is out of the wake of other turbines) and under wake turbulence (when the turbine is in the wake of other turbines). The research questions for this paper are: 
1. Under the same wind conditions, how do ambient and wake turbulence affect the power generation of a wind turbine within a wind plant?

2. For in-wake scenarios, how does the turbulence affect the estimation of wind power when the source of the wind speed is local or remote?

A methodology is developed in this paper to analyze the effects of ambient and wake turbulence on the power generation of a wind turbine. Quantifying different sources of uncertainties in wind power generation attributed to turbulence is the major contribution of this study. Three types of uncertainties in turbine power generation are quantified and analyzed: (i) the uncertainty in power generation with respect to the reported power curve; (ii) the uncertainty in power generation with respect to the estimated power response that accounts for only mean wind speed; and (iii) the uncertainty in power generation with respect to the estimated power response that accounts for both mean wind speed and turbulence intensity. The findings from this study could be utilized to (i) develop more accurate wind power forecasting models, and (ii) design optimal wind plant to maximize the power generation. The uncertainty information would also be uniquely helpful for developing a probabilistic wind power forecast that can be used in power system operation. Different studies have been performed in the literature to quantify wind power uncertainties at different spatial and temporal scales for optimal power system operations [15-20]. The developed method in this paper can be applied to multiple wind farms within a balancing authority to more accurately quantify the wind power uncertainty, thereby improving the economic and reliability performance of the power system scheduling.

The remainder of the paper is organized as follows: a brief summary of the wind plant monitoring data is provided in Section II; the methodology for turbulence analysis is developed in Section III; and Section IV presents the results and discussion of the case study. Note that, during analysis, the wind speed $v s$. output power data for turbine A07 was found to be erroneous, attributed to the malfunctioning local anemometer. Hence, for all multiple turbine studies, reference to "A01-A09" assumes that A07 is not included.

\section{Wind Plant Monitoring Data}

The monitoring data from a wind plant in Northern Colorado with approximately $300 \mathrm{MW}$ of capacity was analyzed in this paper [21]. The turbines are spread throughout an area of approximately $17 \mathrm{~km}$ by $17 \mathrm{~km}$. There are two meteorological towers at this site. The site map in Fig. 1 shows the relative distances among turbines and meteorological towers. The blue, yellow, and red dots represent 1.5-MW turbines, 1-MW turbines, and meteorological towers, respectively. The 1.5-MW and 1-MW turbines are at heights of $80 \mathrm{~m}$ and $69 \mathrm{~m}$, respectively. The turbines are generally lined up in rows that are perpendicular to the prevailing wind direction (northwest) at the site. The average distance between two turbines in the same row is approximately $320 \mathrm{~m}$, or roughly 5 rotor diameters. The distance among rows ranges from approximately $530 \mathrm{~m}$ to more than $8,900 \mathrm{~m}$. The two meteorological towers measure wind speed (in $\mathrm{m} / \mathrm{s}$ ) and direction (from $0^{\circ}$ to $359^{\circ}$ ) at $50 \mathrm{~m}$ and $80 \mathrm{~m}$ at meteorological tower MET01 and at $50 \mathrm{~m}$ and $69 \mathrm{~m}$ at meteorological tower MET02 [21].

A plant information (PI) system is installed at the wind plant to collect detailed operating information. For each turbine, the collected data include turbine status (availability and online status), rotor speed (rpm), power output $(\mathrm{kW})$, nacelle position (degree), and wind speed from the anemometer on top of each nacelle $(\mathrm{m} / \mathrm{s})$. The output of the entire plant is monitored by the utility's supervisory control and data acquisition (SCADA) system and transmitted to its PI system. The wind speed, direction, barometric pressure, and temperature data from the two meteorological towers are also stored by the PI system.

\section{Methodology Development for Turbulence Analysis}

Using the SCADA data from the wind plant, a methodology was developed to analyze the effects of ambient and wake turbulence on the power generation of a single and multiple wind turbines by observing the following sequences.

\section{Single Turbine - Turbine A10:}

a) One turbine on the edge of the wind plant was selected, and two groups of wind and power generation data were determined: (i) an out-of-wake scenario, a set of data (wind speed, wind direction, and wind turbine power generation) when the turbine directly faces incoming winds; and (ii) an in-wake scenario, a set of data when the turbine is in the wake of other turbines.

b) For each group of data, two surrogate models were developed to represent the power generation (i) as a function of the wind speed and (ii) as a function of the wind speed and turbulence intensity. Regression 
methods can be used for this purpose, and the support vector regression (SVR) method was adopted in this work.

c) The uncertainty in the surrogate models was quantified, which serves as an approximation of the uncertainty in turbulence effects on wind power generation.

\section{Multiple Turbines - Turbines A01 - A09:}

a) A group of nine turbines on the edge of the wind plant were selected, and a set of data (wind speed, direction, and and power generation) were determined for an in-wake scenario.

b) For each turbine, four surrogate models were developed to represent the power generation as a function of: (i) turbine measured wind speed, (ii) MET01 tower wind speed, (iii) turbine measured speed and turbulence intensity, and (iv) MET01 wind speed and turbulence intensity. Similar to the single turbine case, SVR was adopted in this work.

c) Similar to the single turbine study, the uncertainty in the surrogate models was quantified to approximate the turbulence-caused uncertainty effects on wind power generation.

\subsection{Determining Two Groups of Wind and Power Generation Data: In-Wake and Out-of-Wake Scenarios}

With the wind plant SCADA data, the analysis of the in-wake and out-of-wake scenarios could be performed. To investigate the effects of ambient turbulence (out-of-wake scenario), a single turbine (or a group of turbines) on the edge of a wind plant was selected. For the single turbine scenario, a 1.5-MW turbine (A10 in Fig. 1) was analyzed in this study. This turbine A10 is mainly in the wake of turbine A09 in certain wind direction conditions. For the multiple turbines scenario, nine turbines (A01-A09 in Fig. 3(a)) were selected. The data used in the paper included (i) 10-minute wind speed, direction, and power generation data from the turbines during the entire year 2011; (ii) 10-minute wind speed and direction data from the meteorological tower (MET01) during the entire year 2011; and (ii) 1-minute wind speed and direction data from the meteorological tower during the summer of 2011 (July, August, and September).

The wind data were divided into two periods where turbine A10 either faces incoming winds directly or is in the wake of other turbines. This is illustrated in Fig. 2. Figure 2(a) shows the out-of-wake scenario; A10 faces incoming winds directly when the wind direction is within the angle of $\alpha_{\mathrm{A} 10}$. To determine $\alpha_{\mathrm{A} 10}$, two dashed lines (A10-A07 and A10-B26) were created by connecting A10 to turbines A07 and B26. Figure 2(b) illustrates the in-wake scenario; $A 10$ is in the wake of other turbines when the wind direction is within the angle of $\beta_{\mathrm{A} 10}$. The angle of $\beta_{\mathrm{A} 10}$ is $30^{\circ}$, which was determined based on the dashed line A10-A09. For A10, (i) the turbine is only in ambient turbulence when the incoming wind direction is within the bounds: $331^{\circ}<\theta<354^{\circ}$; and (ii) the turbine is in the wake of other turbines when the wind direction is within the bounds: $69^{\circ}<\theta<99^{\circ}$. The parameter $\theta$ is the wind direction; the values $0^{\circ}, 90^{\circ}, 180^{\circ}$, and $270^{\circ}$ indicate incoming winds from the north, east, south, and west, respectively.

For the scenario of multiple turbines, the same incoming wind direction $\left(331^{\circ}<\theta<354^{\circ}\right)$ is used for the outof-wake scenario. Figure 3(b) illustrates the determination of wind direction for the in-wake scenario. The angles $\beta_{\mathrm{A} 05}$ and $\beta_{\mathrm{A} 09}$ are $30^{\circ}$, which are determined based on the dashed lines A06-A05 and A10-A09, respectively. The turbines are under wake influences when the incoming wind direction is within both the angles of $\beta_{\mathrm{A} 05}$ and $\beta_{\mathrm{A} 09}$. For the group of turbines, (i) the turbines are only in ambient turbulence when the incoming wind direction is within the bounds: $331^{\circ}<\theta<354^{\circ}$; and (ii) the turbines are in the wake of other turbines when the incoming wind direction is within the bounds: $249^{\circ}<\theta<275^{\circ}$.

Figure 4 shows the scatter plot of recorded wind speed and turbine power generation for the in-wake and outof-wake scenarios. Figures 4(a) and 4(b) represent wind speed measured from A10 and the meteorological tower MET01, respectively. Circle points represent the power and speed relationship when the turbine is only in ambient turbulence, and triangle points represent the power and speed relationship when the turbine is in the wake of other turbines. The reported power curve from the manufacturer is provided in Fig. 4(c). A wind rose is a graphical tool used by meteorologists to provide a succinct illustration of how the wind speed and wind direction are distributed. Figure 5 shows wind rose diagrams from the whole year 2011 at the wind plant. The wind roses were generated from the 10-minute recorded wind data at MET01. It was observed that winds from the northwest and the southeast dominated throughout the year.

\subsection{Surrogate Modeling: Wind Power as a Function of Wind Speed and Turbulence Intensity}

For each set of data (in-wake and out-of-wake), two surrogate models were developed to represent the turbine(s) power generation (i) as a function of the wind speed only and (ii) as a function of the wind speed and turbulence intensity. The first type of surrogate models were expressed as 


$$
P_{a}=\tilde{f}(U) \quad \text { and } \quad P_{w}=\tilde{f}(U)
$$

where $P_{a}$ and $P_{w}$ were the estimated wind power of A10 (or the nine turbines A01-A09) in the out-of-wake and in-wake scenarios, respectively; and $U$ was the wind speed measured from the anemometer on top of the nacelle (or from the meteorological tower). For the multiple turbines scenario, only the wind speed measured from the meteorological tower MET01 was used.

The second type of surrogate models represented the turbine(s) power generation as a function of the wind speed and turbulence intensity $(T I)$, given by

$$
P_{a I}=\tilde{f}(U, T I) \quad \text { and } \quad P_{w I}=\tilde{f}(U, T I)
$$

where $P_{a I}$ and $P_{w I}$ were the estimated wind power of A10 (or the nine turbines A01-A09) in the out-of-wake and in-wake scenarios, respectively.

\subsubsection{Turbulence Intensity}

The TI is defined as the standard deviation of the wind speed within a short time period divided by the mean wind speed during that time period. In this study, 10-minute turbulence intensity was estimated using the 1minute wind data recorded at the meteorological tower.

$$
T I=\frac{U}{\bar{U}}
$$

where ${ }_{U}$ is the standard deviation of the 1-minute wind speed within a 10-minute period, and $\bar{U}$ represents the mean wind speed at the same location during the same time period.

\subsubsection{Support Vector Regression}

A wide variety of surrogate modeling techniques have been developed in the literature, including [23]: (i) the polynomial response surface model, (ii) Kriging, (iii) radial basis functions, (iv) extended radial basis functions, (v) artificial neural networks, and (vi) support vector regression (SVR). The regression method SVR was chosen to represent the power generation of a wind turbine as a function of the wind speed (and turbulence intensity). The SVR approach provides a unique way to construct smooth, nonlinear regression approximations by formulating the surrogate model construction problem as a quadratic programming problem, which is expressed as [24]:

$$
\tilde{f}(x)=\langle w, \Phi(x)\rangle+b
$$

where $\langle x\rangle\rangle$ denotes the dot product, $w$ is a set of coefficients to be determined, and $(x)$ is a map from the input space to the feature space. To solve the coefficients, we can allow a predefined maximum tolerated error (with respect to the actual function value) at each data point, given by [24]

$$
\left|\tilde{f}\left(x_{i}\right)-f\left(x_{i}\right)\right| \leq \varepsilon
$$

where $f(x)$ is the actual function to be approximated. The flatness of the approximated function can be characterized by $w$. By including slack variables $x_{i}$ to the constraints and a cost function, the coefficient $w$ can be obtained by solving a quadratic programming problem given by [24]

$$
\begin{aligned}
& \text { Min } \frac{1}{2}\|w\|^{2}+C \sum_{i=1}^{n_{p}}\left(\xi_{i}+\xi_{i}^{*}\right) \\
& \text { subject to }
\end{aligned}
$$

$$
\begin{aligned}
& f\left(x_{i}\right)-\tilde{f}\left(x_{i}\right) \leq \varepsilon+\xi_{i} \\
& f\left(x_{i}\right)-\tilde{f}\left(x_{i}\right) \leq \varepsilon+\xi_{i}^{*} \\
& \xi_{i}, \xi_{i}^{*} \geq 0
\end{aligned}
$$


primal form of the quadratic programming problem. In most cases, the dual form with a smaller number of constraints is easier to solve, and it is widely used to define the final form of the approximation. The typical mapping functions allowed are radial basis functions, such as the Gaussian function. In this paper, we use an efficient SVR package, a library for support vector machines, developed by Chang and Lin [24].

\subsection{Wind Speed Distribution}

The wind speed distribution is necessary to quantify the available energy (power density) at a site and to design optimal wind plant configurations. The Multivariate and Multimodal Wind Distribution (MMWD) model [25] can capture the joint variation of wind speed, wind direction, and air density, and also represents multimodally distributed data. The MMWD model was developed based on kernel density estimation. KDE is a nonparametric approach to estimate the probability density function of a random variable. KDE has been widely used in the renewable energy community for wind speed distribution characterization and wind and solar power forecasting [25-27]. The details of the MMWD model can be found in Zhang et al. [25].

\subsection{Uncertainty in the Power Response of Turbines (Under In-Wake and Out-of-Wake Scenarios)}

Turbine manufacturers generally provide a smooth (monotonic) power curve to represent the power generation as a function of the incoming wind speed. However, in practice, the power generated by the turbines could be significantly different from that given by the power curve, with respect to the incoming wind speed recorded at the hub height. This discrepancy can be attributed to the following major factors:

i. Wind shear - the variation of wind with vertical distance from the ground, resulting in nonuniformity of the wind faced by the entire turbine rotor.

ii. Turbulence effects - the power generation depends on both mean wind speed and turbulence.

iii. Turbine reliability - the uncertainty in the turbine performance (aerodynamic and control performance).

It is, however, challenging to uniquely attribute uncertainties to these different sources simply based on the recorded incoming conditions and power generation (in in-field turbine operation). In this paper, the particular focus is on illustrating how the degree of uncertainty in the turbine power generation varies between the in-wake and out-of-wake scenarios. In general, in an in-wake scenario a turbine is exposed to a greater amount of turbulence - a combination of atmospheric boundary layer turbulence and wake-induced turbulence-which was expected to impact the variance in the power response of the turbine. In addition, we have also explored whether the estimated variance in the power generation was lower when it was represented as a function of both mean speed and turbulence intensity of the incoming wind (compared to only the mean wind speed).

The uncertainty in power generation with respect to a published/reported power curve (Fig. 3(c)) can be represented as a distribution of the following error $\left(\mathrm{E}_{\mathrm{pc}}\right)$

$$
E_{p c}=P_{c}(U)-P_{g}(U)
$$

where $P_{g}(U)$ and $P_{c}(U)$ represent the recorded power generation and the expected power generation, respectively, given by the power curve for the same incoming mean wind speed.

The uncertainty in power generation with respect to the estimated power response that accounts for only mean wind speed (Eq. 1) can be represented as a distribution of the following error $\left(E_{p f}\right)$

$$
E_{p f}=P_{g}(U)-P_{f}(U)
$$

where $P_{f}(U)$ represents the power generation of a turbine (as a function of mean wind speed) given by the regression model (Eq. 1) fitted from the recorded data.

The uncertainty in power generation with respect to the estimated power response that accounts for both mean wind speed and turbulence intensity (Eq. 2) can be represented as a distribution of the following errors $\left(E_{p f I}\right)$

$$
E_{p f I}=P_{g I}(U, T I)-P_{f I}(U, T I)
$$

where $P_{f I}(U, T I)$ represents the power generation of a turbine (as a function of mean wind speed and turbulence intensity) given by the regression model (Eq. 2) and $P_{g I}(U, T I)$ represents the actual power generation recorded for the same incoming mean wind speed and turbulence intensity.

The above-defined power generation error values were separately determined for the in-wake and out-ofwake scenarios. The error values were normalized by the turbine rated power value (1.5 MW for the single turbine scenario and 13.5 MW for the multiple turbines scenario). The distribution of the normalized errors was then determined using the kernel density estimation method. 


\section{Results and Discussion - Single Wind Turbine (A10)}

\subsection{Distribution of Wind Speeds}

Figure 6 shows the distribution of wind speeds estimated by the MMWD model. The wind speeds measured from both A10 (the anemometer is located on top of the nacelle) and MET01 in year 2011 were analyzed. It was observed that the wind speed distributions from the all-data in-wake scenarios presented two modes. The wind speed distributions from the two out-of-wake scenarios were practically unimodal. For all three types of wind distributions (using all-data, out-of-wake, and in-wake), the wind distribution measured from MET01 was slightly to the right side of the wind distribution measured from the turbine nacelle. This is because the anemometer is located behind the turbine rotor.

\subsection{Uncertainty in Power Generation with Respect to Reported Power Curve of Turbine A10}

The discrepancy between the recorded power generation (from turbine A10) and the expected power generation given by the power curve for the same incoming mean wind speed was calculated by Eq. 8; the recorded wind speed from MET01 at $80 \mathrm{~m}$ was used. The uncertainty in the turbine power generation with respect to the power curve in Fig. 3(c) was quantified by the distribution of the power discrepancy, which is shown in Fig. 7. The power generation error was normalized by the turbine rated power $(1,500 \mathrm{~kW})$. The mean, standard deviation, skewness, and kurtosis of power generation errors are listed in Table 1. Skewness is a measure of the asymmetry of the probability distribution, and is the third standardized moment. The discrepancy is equal to the expected power generation minus the recorded power generation, and thus a positive skewness leads to an over-expected tail, and a negative skewness leads to an under-expected tail. Kurtosis is a measure of the magnitude of the peak of the distribution. A distribution with a positive kurtosis value is known as leptokurtic, which indicates a peaked (narrow) distribution, whereas a negative kurtosis indicates a flat (wide) data distribution, known as platykurtic. The pronounced peak of the leptokurtic distribution indicates a large number of very small turbine power generation errors [28]. According to standard deviation, there was relatively more uncertainty in the power generation in the in-wake scenario. The negative mean and skewness values for the in-wake scenario indicated that the power curve overall tended to underestimate the wind turbine power generation. The fat left tail on the in-wake scenario showed that there are generally more negative extreme errors from the power curve estimation when the turbine is under wakes.

\subsection{Surrogate Modeling and Uncertainty Quantification for Turbine A10: Power $=f(U)$}

Two surrogate models were developed to represent the wind turbine power generation as a function of the wind speed for the in-wake and out-of-wake scenarios, as shown in Fig. 8(a). The 10-minute data from the entire year 2011 were used for this case. In the figure, the solid and dashed lines represent the surrogates developed using the wind speeds measured from A10; the dotted and dot-dashed lines represent the surrogates developed using the wind speeds measured from MET01. It was observed that the turbine generated different power under the same wind conditions between in-wake and out-of-wake scenarios. It was also seen that the turbine was cut out earlier when turbines are out-of-wake than in-wake, showing that wake effects may affect the response time of turbine's cut out. This observation needs to be investigated further in the future. Figure 8(b) illustrates the difference of power generation as a function of wind speed. By comparing the surrogate models developed using the turbine downstream speeds, we observed that (i) the turbine generated more power in the in-wake scenario than in the out-of-wake scenario, when the wind speed was between approximately $3 \mathrm{~m} / \mathrm{s}$ and $9 \mathrm{~m} / \mathrm{s}$, and greater than $15 \mathrm{~m} / \mathrm{s}$; and (ii) the turbine produced almost the same power in the in-wake and out-of-wake scenarios when the wind speed was between approximately $9 \mathrm{~m} / \mathrm{s}$ and $15 \mathrm{~m} / \mathrm{s}$. In the surrogate models developed using wind speeds measured from MET01, it was observed that A10 generated more power in the in-wake scenario than in the out-of-wake scenario over the full range of wind speeds. The average in-wake power generation (over the wind speed range of $0-25 \mathrm{~m} / \mathrm{s}$ ) is approximately $16.7 \%$ and $10.7 \%$ greater than the average out-ofwake power generation based on the A10 downstream speeds and the MET01 wind speed, respectively. Overall, the results indicated that wake turbulence could be helpful in increasing wind power generation for the analyzed wind turbine A10. It was also observed that, when wind speeds approach the turbine's cut-out speed, the power output starts to drop earlier with the surrogate developed based on the turbine downstream speeds. This may be explained by the stronger gusts above the cut out speed causing cut out control to trip sooner. 
The uncertainty in power generation with respect to the estimated power response that accounts for only mean wind speed is quantified in Fig. 8(c). The figure shows the distribution of fitted power errors between the recorded wind power generation and the surrogate estimations. Both wind speeds measured from the anemometers installed on the turbine and the meteorological tower were analyzed. The fitted power errors were normalized by the rated power of A10, which is $1,500 \mathrm{~kW}$. The mean, standard deviation, skewness, and kurtosis values of the error distributions are listed in Table 2. According to the mean and standard deviation values, there was relatively more uncertainty in the surrogate of the in-wake scenario than that of the out-ofwake scenario, thus there was more uncertainty in turbine power generation in the in-wake scenario. The proposed statistical methods could be combined with high-fidelity models (e.g., large eddy simulation) or more accurate measurements (e.g., lidar and sodar measurements) to more accurately characterize the wake effects, thereby helping better understand the in-wake uncertainty information in the power generation. It was also observed from statistical moments that the surrogate developed using wind speeds measured from A10 was more accurate than the surrogate developed using wind speeds measured from MET01.

\subsection{Surrogate Modeling and Uncertainty Quantification for Turbine A10: Power $=f(U, T I)$}

In the previous section, the surrogate model was developed to represent the turbine power generation as a function of wind speed only. Part of the uncertainty in the developed surrogate can be attributed to the lack of incoming condition characterization-e.g., not considering incoming turbulence intensity. Therefore, another surrogate model was further developed to represent the turbine power generation as a function of wind speed and turbulence intensity. The data from the 2011 summer period (July, August, and September) was used for this case. The 10-minute average turbulence intensity was calculated based on the 1-minute wind speed measured from MET01. First, a surrogate was built to investigate the relationship between the wind speed and the turbulence intensity at MET01, which is shown in Fig. 9. At the studied wind plant, the turbulence intensity was relatively high when the wind speed was fairly low (less than $5 \mathrm{~m} / \mathrm{s}$ ) or high (greater than $20 \mathrm{~m} / \mathrm{s}$ ).

Two surrogate models were developed to represent the turbine power generation as a function of the wind speed and the turbulence intensity for the in-wake and out-of-wake scenarios. The wind speed data measured from both A10 and MET01 were used. The contour plots of the developed surrogates are shown in Fig. 10. The surrogates for the out-of-wake and in-wake scenarios that were developed based on measured wind speeds from A10 are shown in Figs. 10(a) and 10(b), respectively; the surrogates for the out-of-wake and in-wake scenarios that were developed based on measured wind speeds from MET01 are shown in Figs. 10(c) and 10(d), respectively. Figure 10 shows that the turbine power generation is sensitive to both wind speed and turbulence intensity. It was observed that: (i) for the out-of-wake scenario, the power generation was more sensitive to the turbulence intensity at lower wind speeds than at higher wind speeds, and (ii) for the in-wake scenario, the power generation was more sensitive to the turbulence intensity when the wind speed was close to the turbine rated speed. In addition, the power generation changed with the turbulence intensity even when the wind speed was greater than the turbine rated speed.

It is important to note that the power output drops with higher wind speeds as it approaches the turbine's cutout speed due to measurement uncertainty and operating practices. The highest power output is observed at about $12 \mathrm{~m} / \mathrm{s}$ wind speed (close to the turbine's rated wind speed) with a small turbulence intensity value. The power output generally decreases as turbulence intensity increases when the turbine is operating at rated power. By comparing the out-of-wake (Figs. 10(a) and 10(c)) and in-wake scenarios (Figs. 10(b) and 10(d)), we observed that the turbine power generation was more sensitive to turbulence when the turbine was in the wake of other turbines.

The uncertainty in the power generation with respect to the estimated power response that accounts for both mean wind speed and turbulence intensity is quantified in Fig. 11. Both wind speeds measured from the anemometers installed on the turbine and the meteorological tower were analyzed. The fitted power errors were normalized by the rated power of A10, which is $1,500 \mathrm{~kW}$. To compare the uncertainties in the power generation between the cases, $\tilde{P}=f(U)$ and $\widetilde{P}=f(U, T I)$, surrogates of the power generation as a function of the wind speed only was again developed using the A10 and MET01 data during the 2011 summer. Table 3 lists the mean, standard deviation, skewness, and kurtosis values of the error distributions. According to the standard deviation values, the uncertainty in the surrogate model was reduced by considering the turbulence intensity. For surrogates developed using wind speed measured from the turbine, the uncertainty levels in Fig. 11(a) were not significantly different; for surrogates developed using wind speed measured from the meteorological tower as shown in Fig. 11(b), the uncertainty in the out-of-wake scenario was significantly lower than that in the in-wake scenario. For surrogates with meteorological tower data, the standard deviation of the out-of-wake scenario is approximately $52 \%$ less than that of the in-wake scenario when considering only wind speed. The number increases to and 54\% when considering both wind speed and turbulence intensity. It was again observed from 
the statistical moments that the surrogate developed using wind speeds measured from A10 was more accurate than the surrogate developed using wind speeds measured from MET01. The model based on MET tower data model causes a much large error for the in-wake estimates, indicating MET tower data is not sufficient to capture and illustrate the wake effects, though the MET tower is close to the analyzed turbine.

\section{Results and Discussion - Multiple Wind Turbines (A01-A09)}

\subsection{Surrogate Modeling and Uncertainty Quantification for the Multiple Turbines Scenario}

Two types of surrogate models, Power $f(U)$ and $\operatorname{Power}=f(U, T I)$, were developed for both the in-wake and out-of-wake scenarios. Because the wind speeds measured from the anemometers of the nine turbines are different, only the wind speed data measured from MET01 were used for this scenario. The same 10-minute average turbulence intensity calculated based on MET01 was also adopted in this scenario. The contour plots of the developed surrogates $($ Power $=f(U, T I))$ for the out-of-wake and in-wake scenarios are shown in Fig. 12. It was observed that, for both out-of-wake and in-wake scenarios, the power generation was more sensitive to the turbulence intensity with lower wind speeds than with higher wind speeds. As in the single turbine scenario, we observed that: (i) power generation changed with turbulence intensity even when wind speed was greater than the turbine's rated speed; and (ii) turbine power generation was more sensitive to turbulence for the in-wake scenario.

Figure 13 shows the uncertainty in the power generation for the two types of surrogate models $\operatorname{Power}=f(U)$ and Power $=f(U, T I)$. The fitted power errors were normalized by the rated power of the nine turbines, which is $13,500 \mathrm{~kW}$. It was observed that: (i) the uncertainty in the out-of-wake scenario was significantly lower than that in the in-wake scenario; (ii) for the in-wake scenario, the uncertainty levels between the Power $=f(U)$ and Power $=f(U, T I)$ were not significantly different; and (iii) for the out-of-wake scenario, the uncertainty level was relatively lower when considering the turbulence intensity in the surrogate model. These observations could help wind farm operators to optimally determine strategies for reducing wind power uncertainty attributed to turbulence, such as applying different control set points based on wind speed and turbulence intensity, and separately optimizing parameters of wind turbine controllers between in-wake and out-of-wake scenarios.

\subsection{Power and Uncertainty Reduction for the Multiple Turbines Scenario}

This section focuses on the uncertainty quantification for the multiple turbines scenario. As described before, the 10-min resolution wind speed data was available for an entire year, and the 1-min high resolution data was only available for the summer.

\subsubsection{Low-resolution data analysis for the entire year}

This section analyzes the effects of data source (e.g., resolution and measured location) on the wind power uncertainty estimation. Considering that only the lower resolution data is available at MET tower and wind turbines, turbulence intensity is not considered in this case.

Estimation errors for turbines w.r.t. only their individual speeds: The uncertainty in the power estimation for all the turbines is shown in Fig. 14(a). Note that, the error distribution is not spread out on either side of ' 0 ', compared to Fig. 13. This indicates that the use of turbine's own measured wind speed improves their power estimation accuracy, which is expected.

Estimation errors for turbines w.r.t. only MET tower speed: Figure 14(b) shows the distribution of power estimation error when using MET tower measurement. Note that the error distribution is much more spread out along the x-axis, compared to Fig. 14(a). This reiterates the observation that, though MET tower wind speed data can be used for wind power output estimation, it generally introduces more uncertainty than the local turbine measured data. However, MET tower measurements generally have higher resolution data, from which the turbulence intensity can be calculated and used for the uncertainty quantification. In addition, MET tower data can also be used to calibrate the wind speed and direction measurements from different turbines.

\subsubsection{High-resolution data analysis for the summer months}

This section analyzes the effects of data source (resolution and measured location) on the wind power uncertainty estimation using high resolution MET tower data for summer months. Turbulence intensity is considered in this case. Both the contour plots for surrogate models and the uncertainty plots are presented. 
Estimation errors for turbines A01-09 w.r.t. their individual speed and TI: It is observed that the use of turbulence intensity has greatly reduced the uncertainty (Fig. 15(b)) compared to Fig. 14(a). This can be attributed to the better turbulence characterization (by including TI) and the use of local turbine measured wind speeds, which are highly correlated to the turbine's output. Both the single turbine (in Section 4) and multiple turbines scenarios have shown that, the uncertainty in wind power generation estimation can be reduced by considering the turbulence intensity. Turbulence intensity could possibly be utilized as a parameter for wind power forecasting and wind farm operations.

Estimation errors for turbines A01-09 w.r.t. MET tower speed and TI: When relatively less correlated MET tower wind speed data was used, the turbulence-caused uncertainty in wind power generation increased (Fig. 16 (b)) in contrast to Fig. 14(a). Overall, the power estimation is highly sensitive to the source of the collected wind speed data.

\subsubsection{Power curve estimation}

As another measure to quantify the accuracy of the wind power estimation, the developed surrogate model is also used to generate the power curve for the corresponding turbines. This helps in measuring the relative performance of various techniques used, and gives a visual understanding of the accuracy. The metrics of root mean square error (RMSE) and mean absolute error (MAE) are calculated. Interesting patterns are observed in Fig. 17. Four cases are presented in Fig. 17 based on the input data, which present the turbine power output estimation as:

i) Case 1: A function of turbine's own wind speed

ii) Case 2: A function of MET tower wind speed

iii) Case 3: A function of turbine's own wind speed and calculated TI

iv) Case 4: A function of MET tower wind speed and calculated TI

It is seen that, both RMSE and MAE present the same trend in Fig. 17. Errors are significantly smaller, when using the turbine's speed to estimate its output power (Case 1). This is because the wind data is strongly correlated to the respective turbine. Using the MET tower data (Case 2) reduces the accuracy of power estimation. This is due to the relatively weak correlation between the MET tower data and turbine's output power. The most accurate power estimation (least errors) is obtained when using turbine's own speed together with the TI calculated using the high resolution summer MET tower data (Case 3). Most interestingly, errors increase significantly, when using the MET tower wind speed data and TI together (Case 4), due to the weak correlation between the MET tower data and turbine's output power.

Power curve estimation using the surrogate models: Power curve is the most important metric used to in turbine selection for wind plant design. To enable an automatic turbine type selection in wind farm optimization, approximated turbine curves expressed as a mathematic function is needed to form the optimization problem [10]. In this analysis, power curves were estimated using the surrogate models generated for Case 1 . These estimated power curves were compared to the original power curve as given by the manufacturer (Fig. 4(c)). RMSE and MAE quantify the deviation of the estimated power curve, as presented in Fig. 18. It is seen that, the MAE of all the turbines is between $100 \mathrm{~kW}$ and $200 \mathrm{~kW}$, indicating that the estimates are relatively close to the expected outputs in general.

\section{Conclusions}

This paper developed a methodology to analyze how ambient and wake turbulence affect the power generation of a wind turbine. The monitoring wind speed, wind direction, and power generation SCADA data from a wind plant in Northern Colorado were used for the analysis. For the analyzed in-wake and out-of-wake scenarios, surrogate models were developed to represent the turbine power generation, (i) as a function of the wind speed and (ii) as a function of the wind speed and turbulence intensity. Uncertainties in the surrogate models and thereby in the turbine power generation were quantified.

We found that in the same wind conditions the turbine generated different power between the in-wake and the out-of-wake scenarios, and the turbine generally produced more power in the in-wake scenario. In the surrogate model that represented the power generation as a function of wind speed and turbulence intensity, we found that (i) in the out-of-wake scenario, for both the single turbine and multiple turbines cases, the power generation was more sensitive to the turbulence intensity with lower wind speeds than with higher wind speeds; and (ii) in the in-wake of the single turbine scenario, the power generation was more sensitive to the turbulence intensity when the wind speed was close to the turbine rated speed. The uncertainty quantification results generally showed that more uncertainty in the power generation was present in the in-wake scenario than in the out-of-wake scenario. 
The results of this paper could be useful in wind power forecasting models that consider the output of an entire plant. The uncertainty in the plant output would be much higher when the wind direction is such that a large number of turbines are in the wake of other turbines. The uncertainty information would be uniquely helpful for developing a probabilistic wind power forecast. It also speaks to the importance of providing many sources of wind speed data to the forecasting algorithms, as there is clear value from using individual turbine wind speed measurements for the power conversion process. Thus, utilizing the individual turbine wind speed measurements in forecasting applications should yield better results than MET tower data alone. The uncertainty analysis could also be useful for the optimal design of a wind plant, by maximizing the power generation of wind turbines that are under wakes. This could be especially useful for the design of large plants, or plants in locations where there is not a single predominate wind direction.

\section{Acknowledgements}

This work was supported by the U.S. Department of Energy under Contract No. DE-AC36-08-GO28308 with the National Renewable Energy Laboratory.

\section{References}

[1] WWEA, World wind energy report 2013, Technical Report, World Wind Energy Association, Bonn, Germany, April 2014.

[2] Barthelmie, R. J., and Pryor, S. C., "An Overview of Data for Wake Model Evaluation in the Virtual Wakes Laboratory," Applied Energy, Vol. 104, 2013, pp. 837-844.

[3] Barthelmie, R. J., Larsen, G. C., Frandsen, S. T., Folkerts, L., Rados, K., Pryor, S. C., Lange, B., and Schepers, G., "Comparison of Wake Model Simulations with Offshore Wind Turbine Wake Profiles Measured by Sodar," Journal of Atmospheric and Oceanic Technology, Vol. 23, No. 7, 2006, pp. 888-901.

[4] Frandsen, S., and Thøgersen, M. L., "Integrated Fatigue Loading for Wind Turbines in Wind Farms by Combining Ambient Turbulence and Wakes," Wind Engineering, Vol. 23, No. 6, 1999, pp. 327-339.

[5] Newman, J., Lebron, J., Meneveau, C., and Castillo, L., "Streamwise Development of the Wind Turbine Boundary Layer over A Model Wind Turbine Array," Physics of Fluids, Vol. 25, No. 8, 2013, pp. 085108.

[6] Makridis, A., and Chick, J., "Validation of A CFD Model of Wind Turbine Wakes with Terrain Effects," Journal of Wind Engineering and Industrial Aerodynamics, Vol. 123, 2013, pp. 12-29.

[7] Gaumond, M., Réthoré, P. E., Ott, S., Peña, A., Bechmann, A., and Hansen, K. S., "Evaluation of the Wind Direction Uncertainty and Its Impact on Wake Modeling at the Horns Rev Offshore Wind Farm," Wind Energy, 2013, DOI: 10.1002/we.1625.

[8] Tong, W., Chowdhury, S., Zhang, J., and Messac, A., "Impact of Different Wake Models On the Estimation of Wind Farm Power Generation," 14th AIAA/ISSMO Multidisciplinary Analysis and Optimization Conference, Indianapolis, Indiana, September 17-19, 2012.

[9] Chowdhury, S., Zhang, J., Messac, A., and Castillo, L., "Unrestricted Wind Farm Layout Optimization (UWFLO): Investigating Key Factors Influencing the Maximum Power Generation," Renewable Energy, Vol. 38, No. 1, 2012, pp. 16-30.

[10] Chowdhury, S., Zhang, J., Messac, A., and Castillo, L., "Optimizing the Arrangement and the Selection of Turbines for Wind Farms Subject to Varying Wind Conditions," Renewable Energy, Vol. 52, 2013, pp. 273-282.

[11] Messac, A., Chowdhury, S., and Zhang, J., "Characterizing and Mitigating the Wind Resource-based Uncertainty in Farm Performance," Journal of Turbulence, Vol. 13, Issue 13, 2012, pp. 1-26.

[12] Al-Shammari, E.T., Shamshirband, S., Petković, D., Zalnezhad, E., Yee, L., Taher, R.S., and Ćojbašić, Ž., "Comparative Study of Clustering Methods for Wake Effect Analysis in Wind Farm," Energy, Vol. 95, 2016, pp. 573-579.

[13] Nikolić, V., Shamshirband, S., Petković, D., Mohammadi, K., Ćojbašić, Ž., Altameem, T.A., and Gani, A., "Wind Wake Influence Estimation on Energy Production of Wind Farm by Adaptive Neuro-Fuzzy Methodology," Energy, Vol. 80, 2015 , pp. 361-372.

[14] Kuo, J.Y., Romero, D.A., and Amon, C.H., "A Mechanistic Semi-Empirical Wake Interaction Model for Wind Farm Layout Optimization," Energy, Vol. 93, 2015, pp. 2157-2165.

[15] Sedghi M., Ahmadian A., and Aliakbar-Golkar M., "Optimal Storage Planning in Active Distribution Network Considering Uncertainty of Wind Power Distributed Generation,” IEEE Transactions on Power System, Vol. 31, No. 1, 2016 , pp. $304-316$.

[16] Sedghi M., Ahmadian A., Pashajavid E., and Aliakbar-Golkar M., "Storage Scheduling for Optimal Energy Management in Active Distribution Network Considering Load, Wind, and Plug-In Electric Vehicles Uncertainties," Journal of Renewable and Sustainable Energy, Vol. 7, No. 3, 2015, pp. 033120.

[17] Alavi S. A., Ahmadian A., Aliakbar-Golkar M., "Optimal Probabilistic Energy Management in A Typical Micro-Grid Based-on Robust Optimization and Point Estimate Method," Energy Conversion and Management, Vol. 95, 2015, pp. 314-325.

[18] Zhang, J., Hodge, B.-M., Miettinen, J., Holttinen, H., Gomez-Lazaro, E., Cutululis, N., Litong-Palima, M., Sorensen, P., Lovholm, A. L., Berge, E., and Dobschinski, J., "Analysis of Variability and Uncertainty in Wind Power Forecasting: An International Comparison," 12th International Workshop on Large-Scale Integration of Wind Power into Power Systems as well as on Transmission Networks for Offshore Wind Power Plants, Paper No. WIW13-1115, London, UK, October 22-24, 2013.

[19] Wang, J., A. Botterud, R. Bessa, H. Keko, L. Carvalho, D. Issicaba, J. Sumaili, and V. Miranda, "Wind Power Forecasting Uncertainty and Unit Commitment," Applied Energy, Vol. 88, No. 11, 2011, pp. 4014-4023.

[20] Gass, V., F. Strauss, Johannes Schmidt, and E. Schmid, "Assessing The Effect of Wind Power Uncertainty on Profitability," Renewable and Sustainable Energy Reviews, Vol. 15, No. 6, 2011, pp. 2677-2683.

[21] Wan, Y. H., Ela, E., and Orwig, K., "Development of an Equivalent Wind Plant Power-Curve," WindPower 2010, Dallas, Texas, May 23-26, 2010.

[22] GE Energy, “1.5-MW Wind Turbine Brochure,” http://www.ge-energy.com/. 
[23] Zhang, J., Chowdhury, S., and Messac, A., “An Adaptive Hybrid Surrogate Model,” Structural and Multidisciplinary Optimization, Vol. 46, No. 2, 2012, pp. 223-238.

[24] Chang, C. C., Lin, C. J., "LIBSVM: A Library for Support Vector Machines," ACM Transactions on Intelligent Systems and Technology, Vol. 2, No. 3, 2011, pp. 27:1-27:27.

[25] Zhang, J., Chowdhury, S., Messac, A., and Castillo L., "A Multivariate and Multimodal Wind Distribution Model," Renewable Energy, Vol. 51, 2013, pp. 436-447.

[26] Zhang J, Hodge B, Florita A. Joint Probability Distribution and Correlation Analysis of Wind and Solar Power Forecast Errors in the Western Interconnection. Journal of Energy Engineering, Vol. 141, 2015, pp.B4014008.

[27] Juban J, Siebert N, Kariniotakis GN. Probabilistic short-term wind power forecasting for the optimal management of wind generation. IEEE Power Eng. Society, Lausanne Power Tech Conf. Proc., 2007: 683-688.

[28] Zhang, J., Florita, A., Hodge, B.-M., Lu, S., Hamann, H. F., Banunarayanan, V., Brockway, A. M., "A Suite of Metrics for Assessing the Performance of Solar Power Forecasting," Solar Energy, Vol. 111, 2015, pp. 157-175.

Table 1. Statistical moments of power generation errors with respect to the reported power curve

\begin{tabular}{lcccc}
\hline \multicolumn{1}{c}{ Scenario } & Mean & Standard deviation & Skewness & Kurtosis \\
\hline Out-of-wake & 0.026 & 0.114 & -1.625 & 5.679 \\
In-wake & -0.069 & 0.164 & -2.017 & 5.453 \\
\hline
\end{tabular}

Table 2. Statistical moments of fitted power generation errors in the case of Power $=\mathrm{f}(\mathrm{U})$

\begin{tabular}{|c|c|c|c|c|c|}
\hline Anemometer location & Scenario & Mean & Standard deviation & Skewness & Kurtosis \\
\hline \multirow{2}{*}{ Wind speed measured from turbine } & Out-of-wake & 0.002 & 0.047 & 2.526 & 29.191 \\
\hline & In-wake & 0.001 & 0.067 & 4.687 & 82.847 \\
\hline \multirow{2}{*}{ Wind speed measured from MET01 } & Out-of-wake & 0.020 & 0.108 & 2.362 & 9.505 \\
\hline & In-wake & 0.038 & 0.162 & 2.339 & 6.460 \\
\hline
\end{tabular}

Table 3. Statistical moments of fitted power generation errors in the cases of Power $=\mathrm{f}(\mathrm{U})$ and Power $=\mathrm{f}(\mathrm{U}, \mathrm{TI})$

\begin{tabular}{clcccc}
\hline Anemometer location & \multicolumn{1}{c}{ Scenario } & Mean & Standard deviation & Skewness & Kurtosis \\
\hline \multirow{3}{*}{ Wind speed measured from } & Out-of-wake (speed only) & 0.007 & 0.045 & 1.307 & 1.609 \\
turbine & In-wake (speed only) & 0.007 & 0.050 & 2.590 & 18.388 \\
& Out-of-wake (speed and TI) & 0.007 & 0.044 & 1.390 & 1.833 \\
& In-wake (speed and TI) & 0.004 & 0.048 & 2.892 & 22.774 \\
Wind speed measured from & Out-of-wake (speed only) & 0.031 & 0.095 & 1.747 & 2.855 \\
MET01 & In-wake (speed only) & 0.069 & 0.196 & 1.201 & 0.473 \\
& Out-of-wake (speed and TI) & 0.020 & 0.086 & 1.676 & 3.832 \\
& In-wake (speed and TI) & 0.042 & 0.186 & 1.167 & 1.075 \\
\hline
\end{tabular}




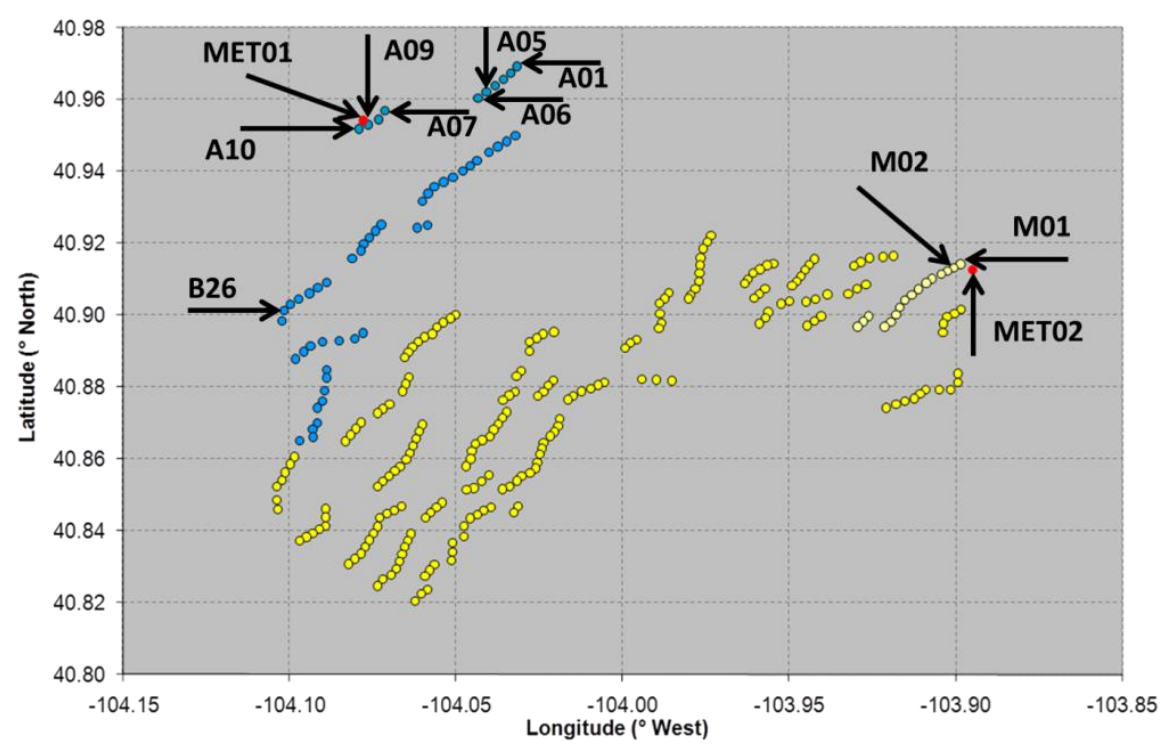

Figure 1. Turbine and met tower locations [14]

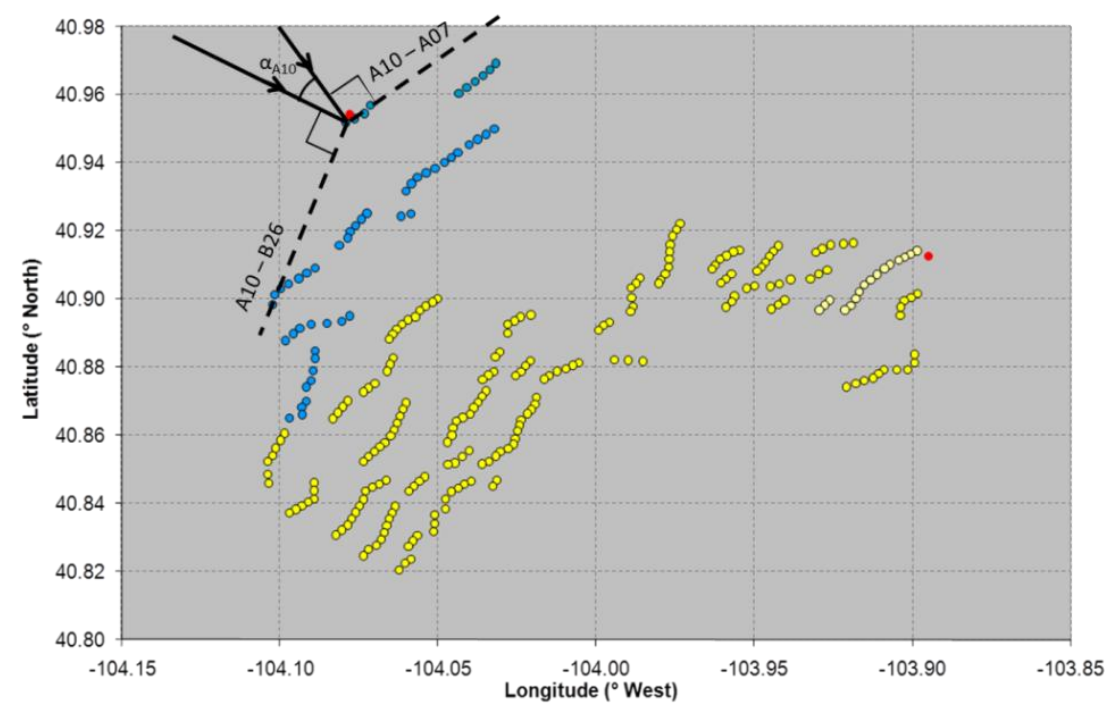

(a) Determining $\alpha_{\mathrm{A} 10}$ (out-of-wake scenario)

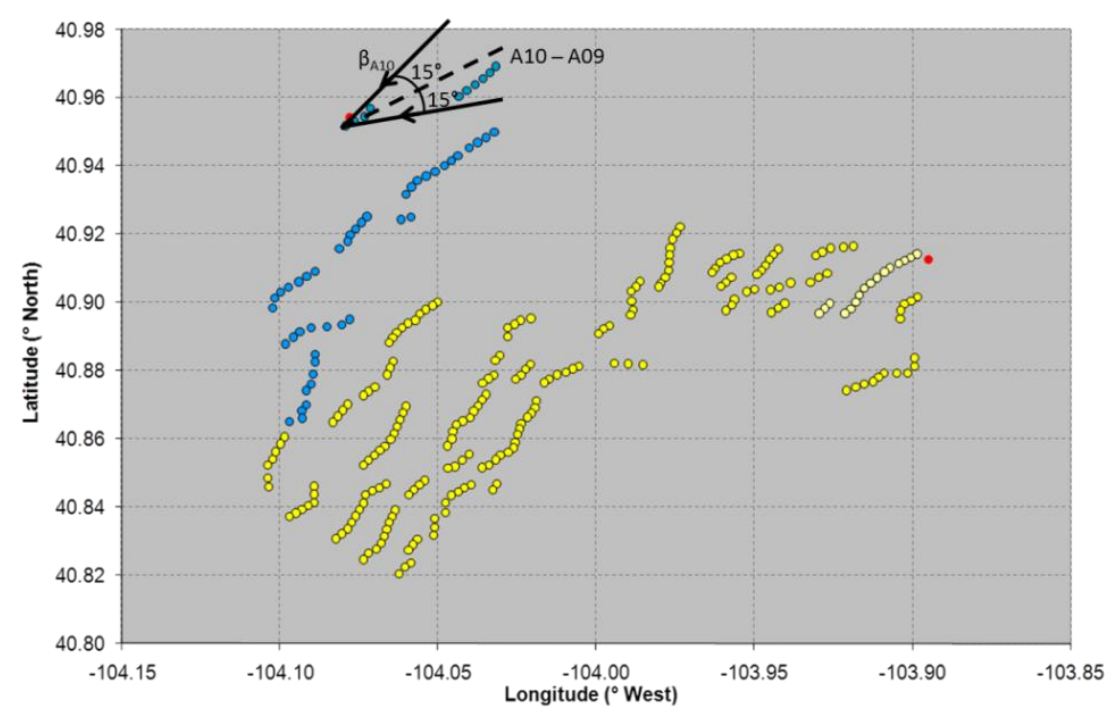

(b) Determining $\beta_{\mathrm{A} 10}$ (in-wake scenario)

Figure 2. Determining the two groups of data for turbulence analysis (turbine A10) 


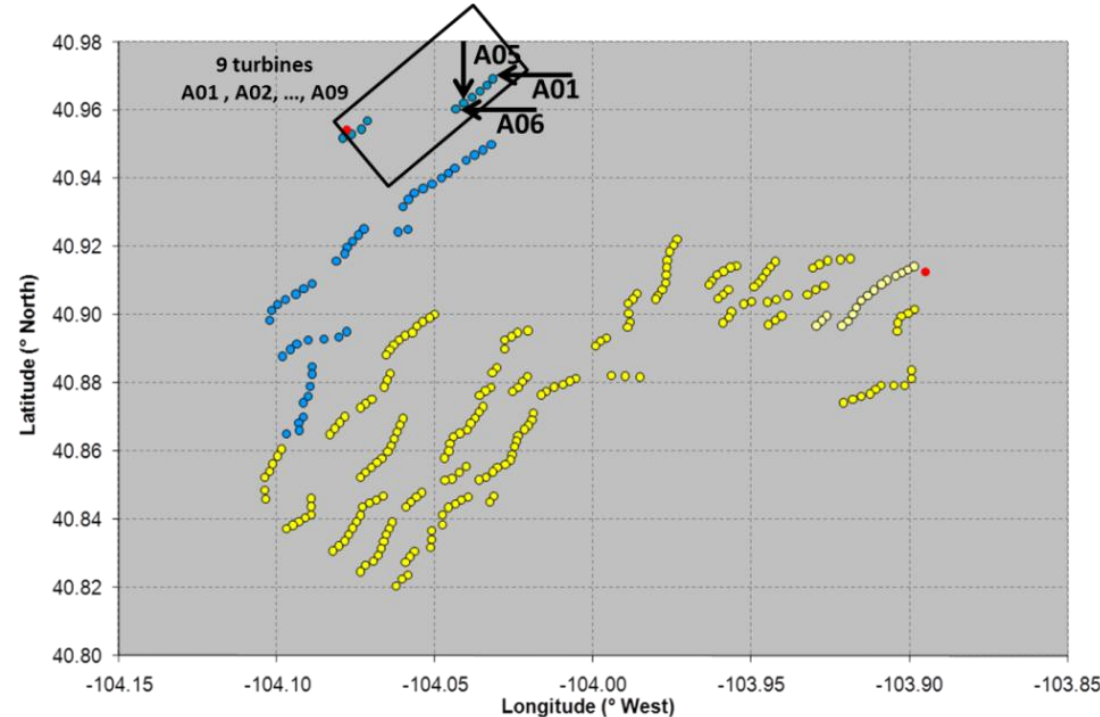

(a) Determining the turbine group A01-A09

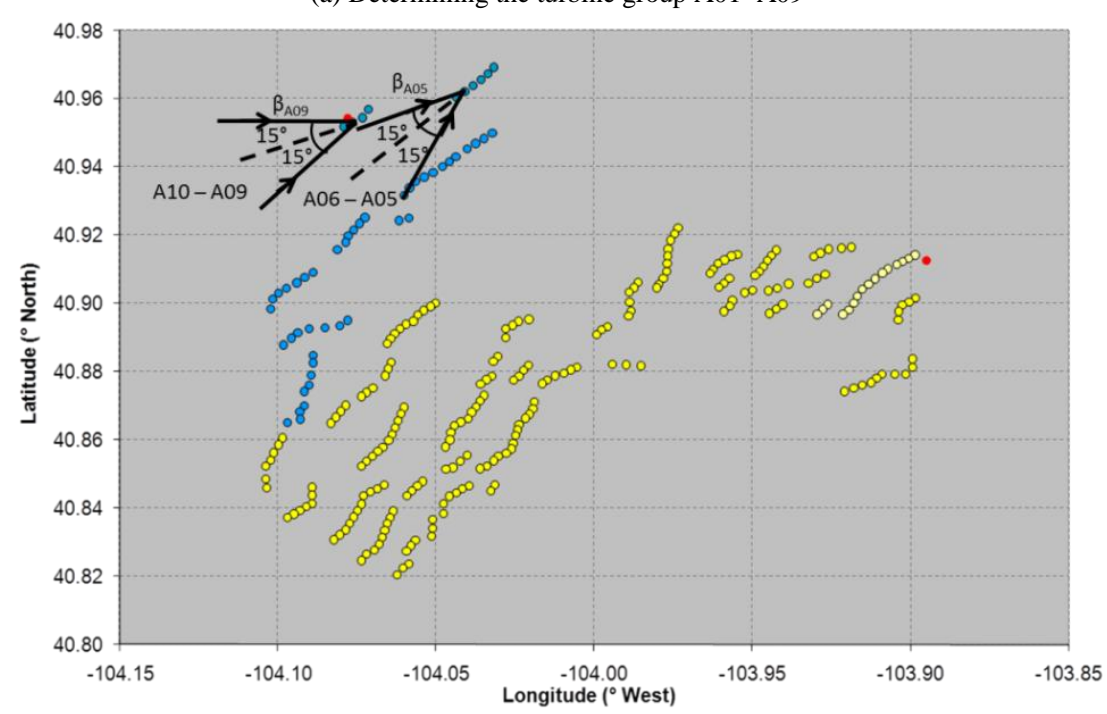

(b) Determining wind directions for the in-wake scenario

Figure 3. Determining the two groups of data for turbulence analysis (turbine group A01-A09)

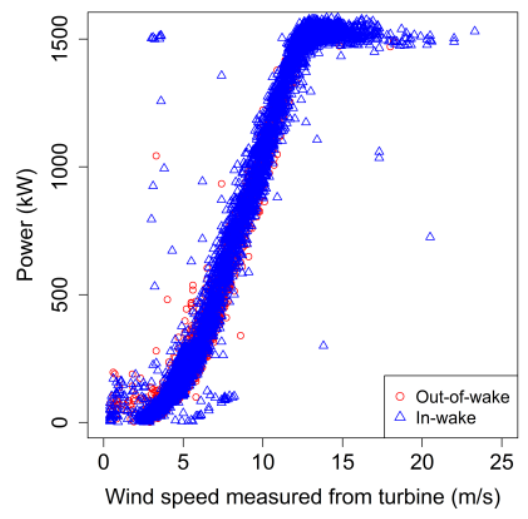

(a) Wind speed measured from turbine

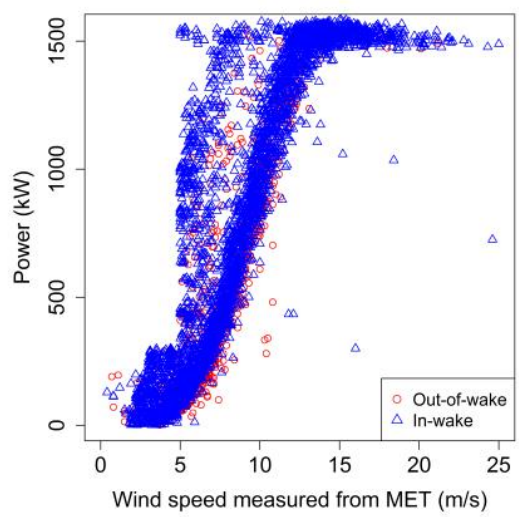

(b) Wind speed measured from MET01

(c) Power curve reported by manufacturer [22]

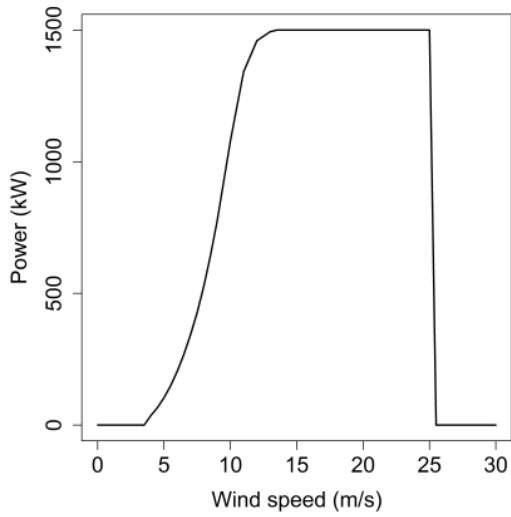

A 0 


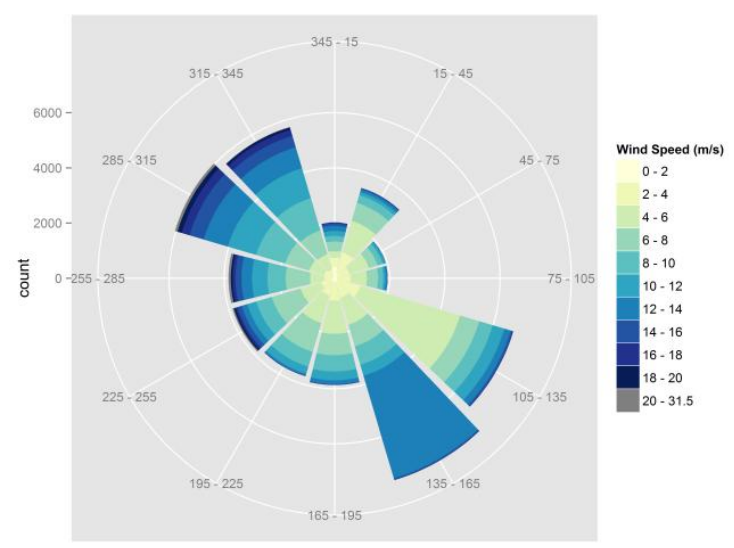

(a) 80 meters

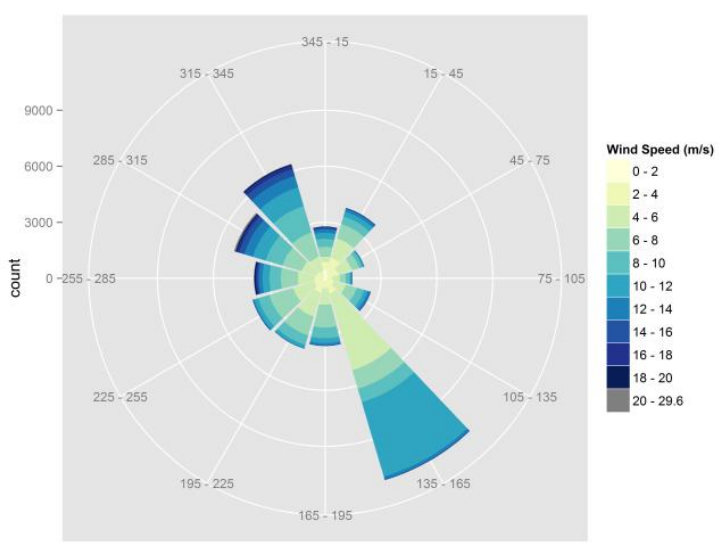

(b) 50 meters Figure 5. Wind rose diagrams at the meteorological tower MET01

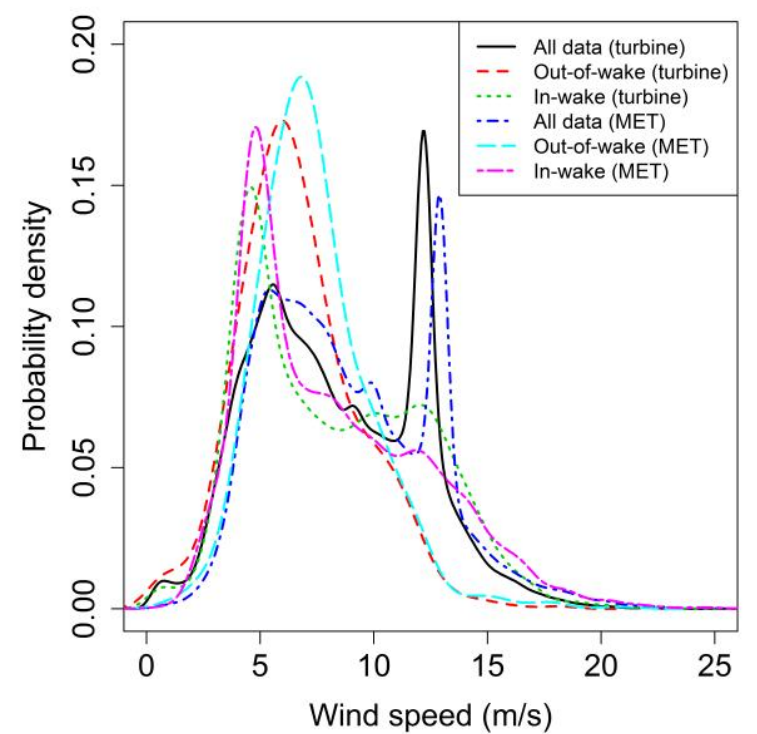

Figure 6. Distribution of wind speeds

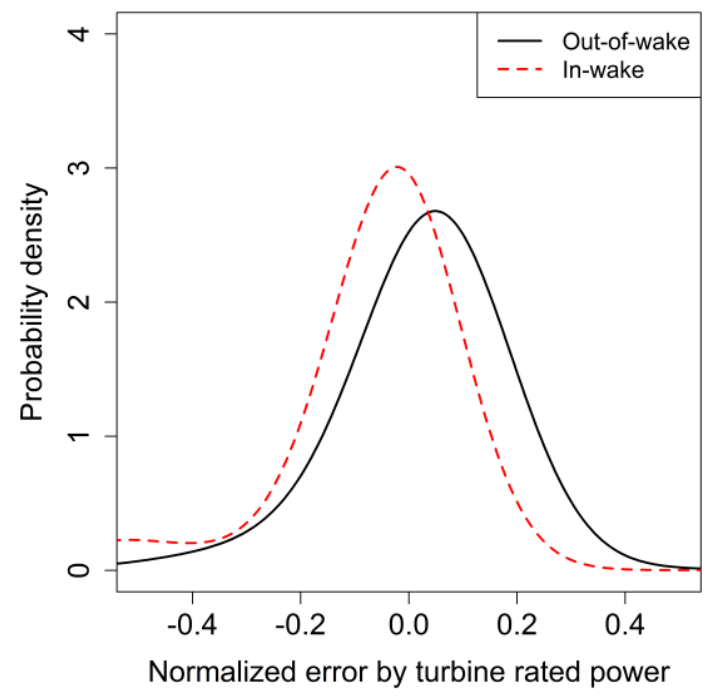

Figure 7. Uncertainty in power generation with respect to the reported power curve 


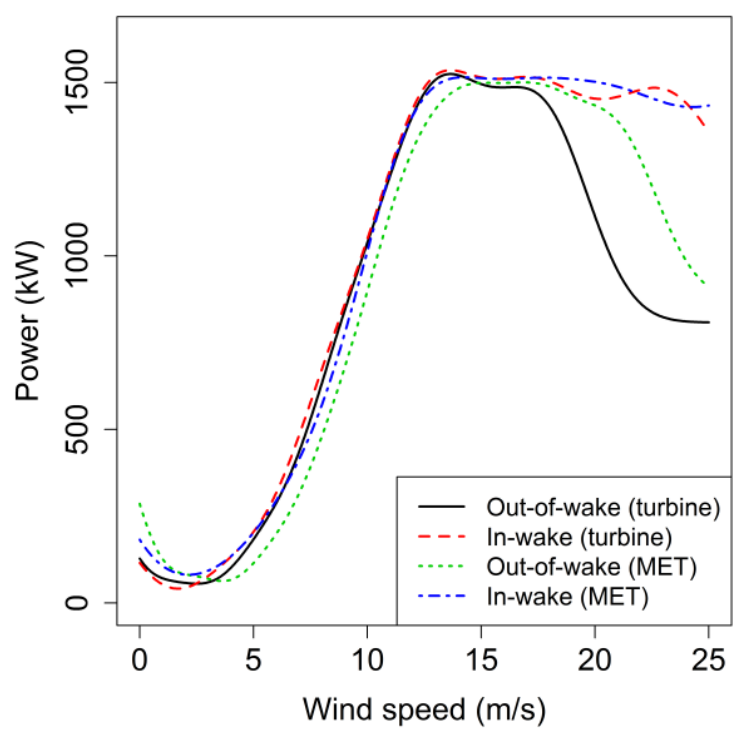

(a) Surrogate models

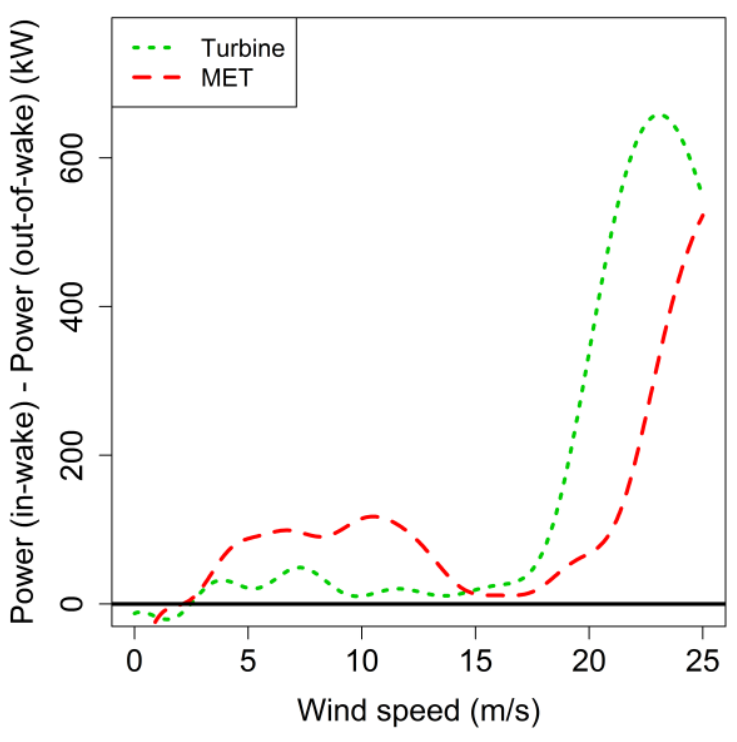

(b) Distributions of fitted power errors

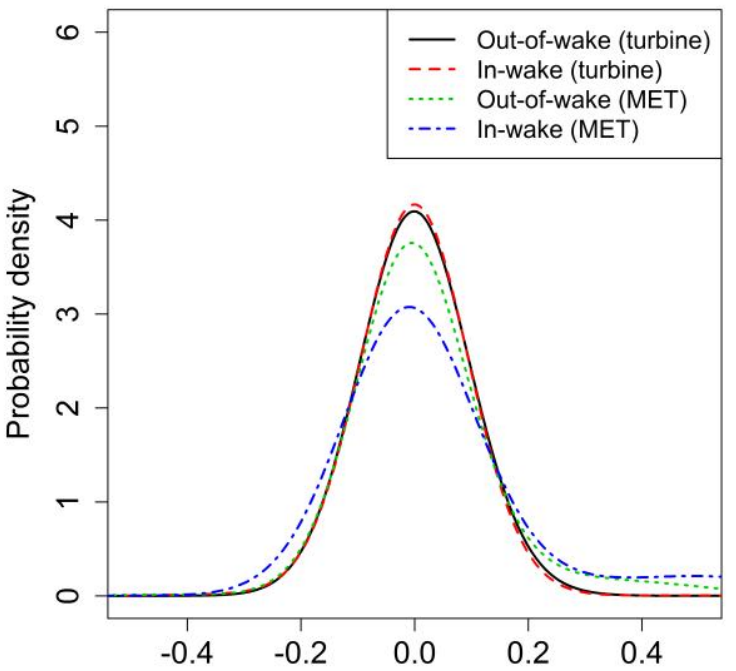

Normalized error by turbine rated power

(c) Distributions of fitted power errors

Figure 8. Surrogate modeling and uncertainty quantification in the case of Power=f(U)

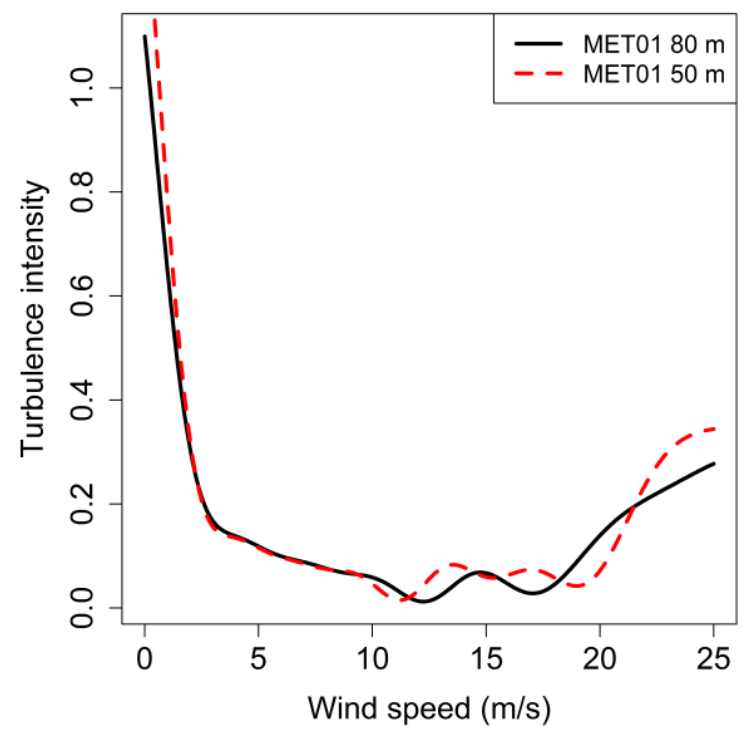




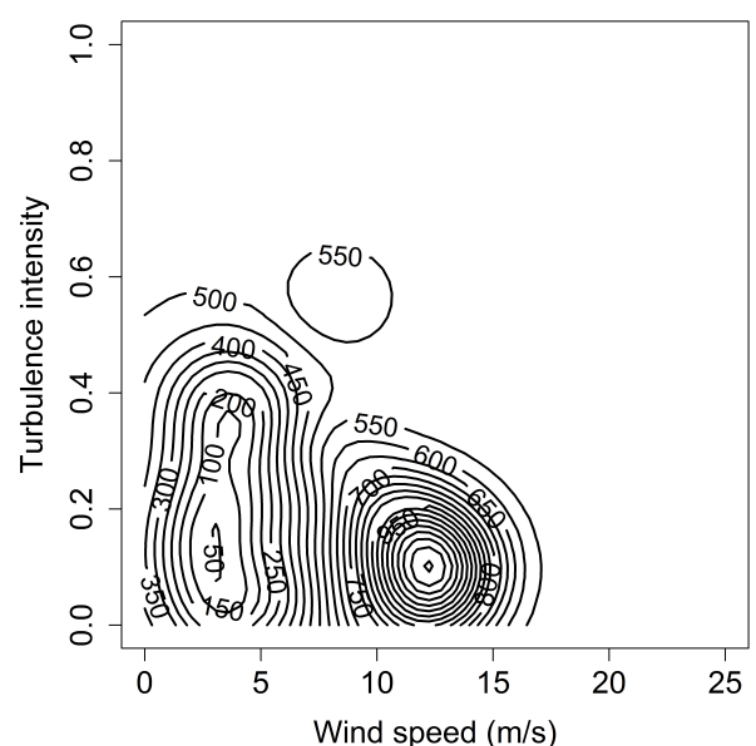

(a) Surrogate with turbine speed (out-of-wake)

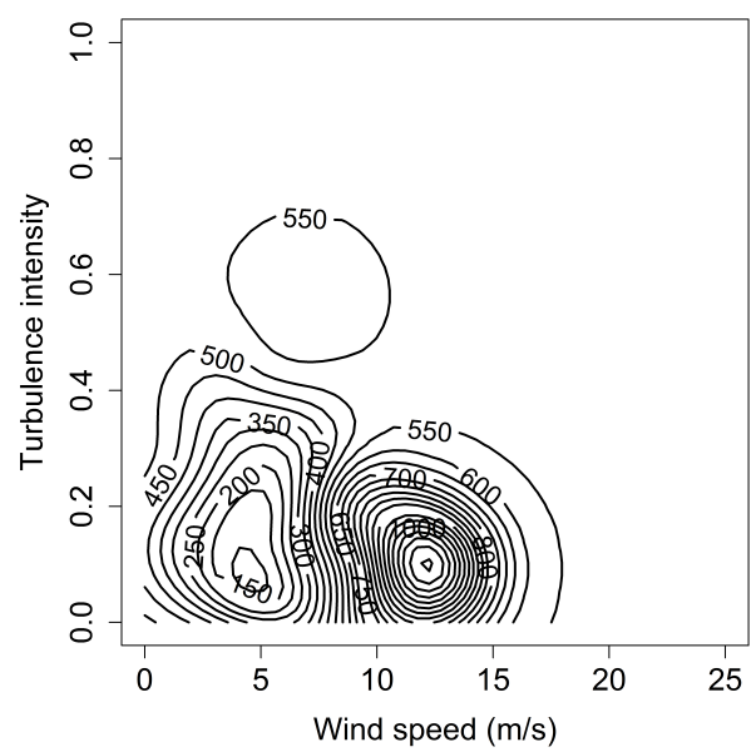

(c) Surrogate with MET01 speed (out-of-wake)

Figure 10. Surrogate modeling in the case of Powe)

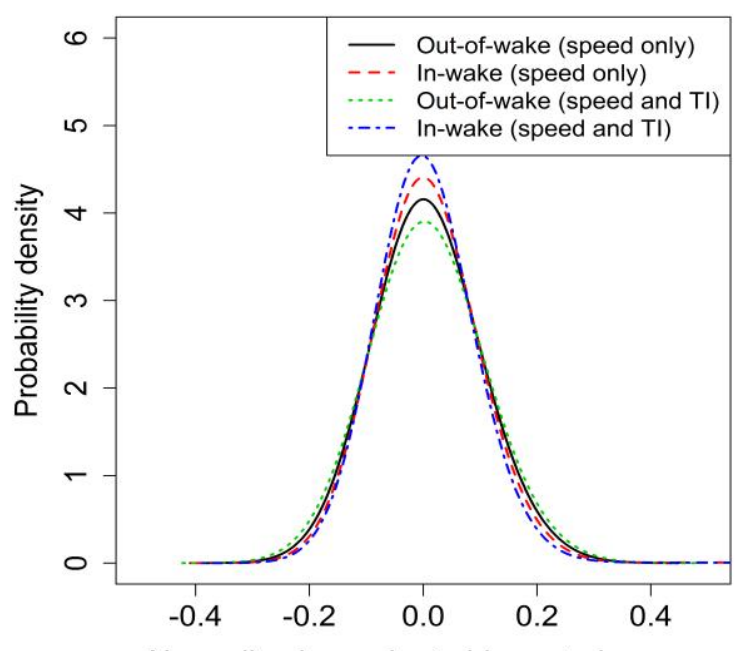

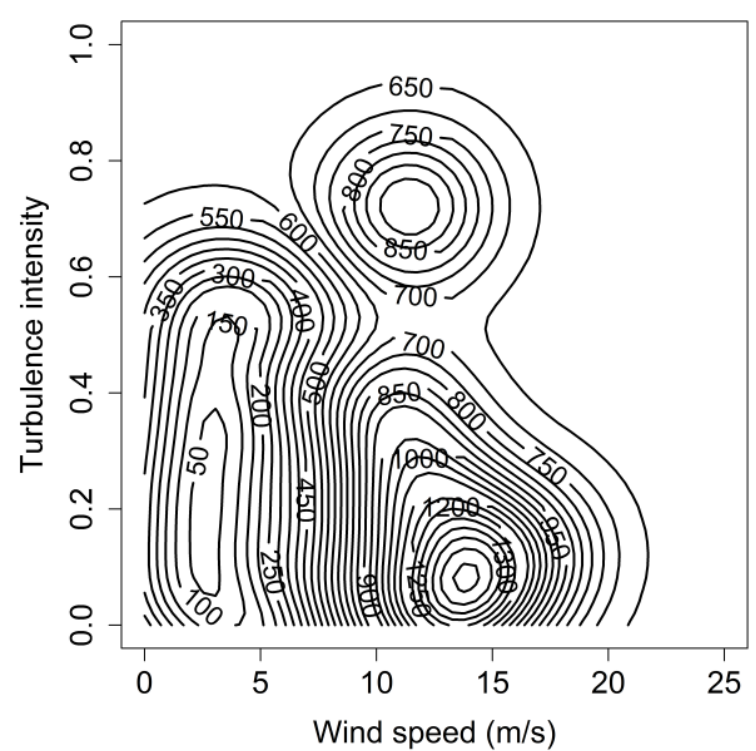

(b) Surrogate with turbine speed (in-wake)

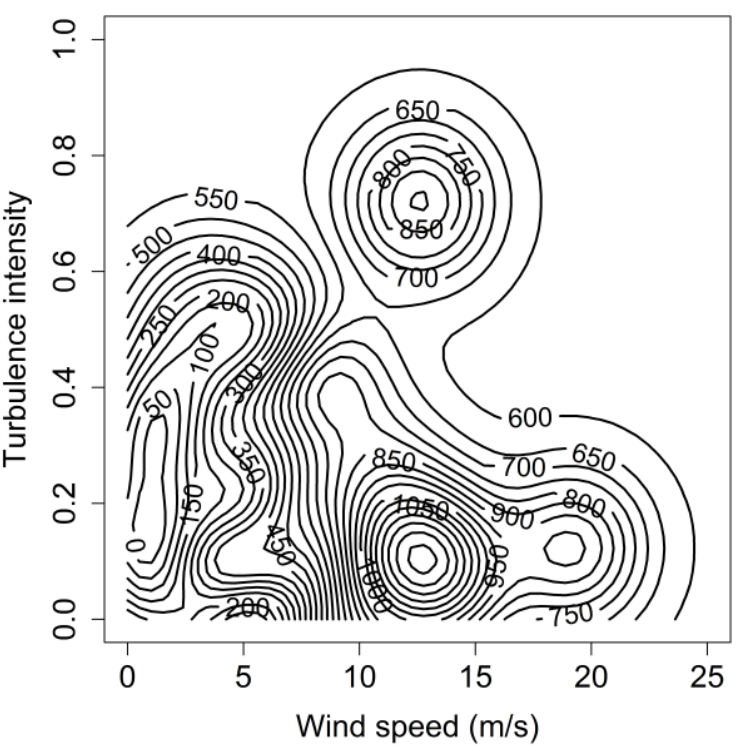

(d) Surrogate with MET01 speed (in-wake)

TI); the numbers shown in the figures are the wind power

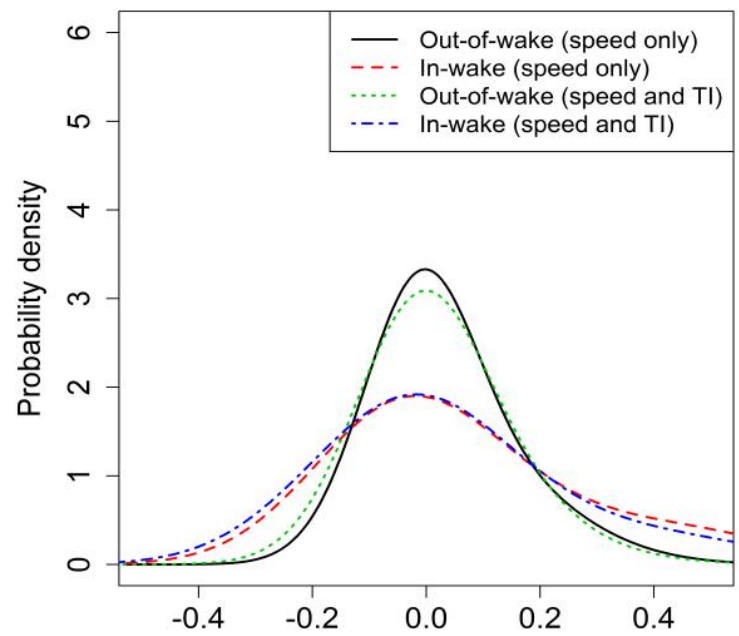

Normalized error by turbine rated power (b) Surrogate with speeds measured from MET01 
Figure 11. Uncertainty quantification in the case of Power $=f(U, T I)$

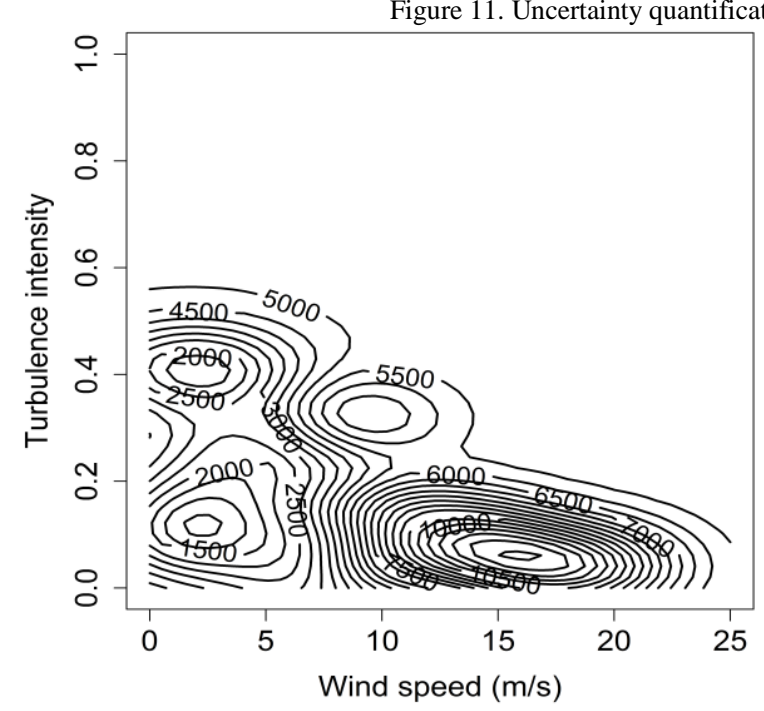

(c) Surrogate with MET01 speed (out-of-wake)

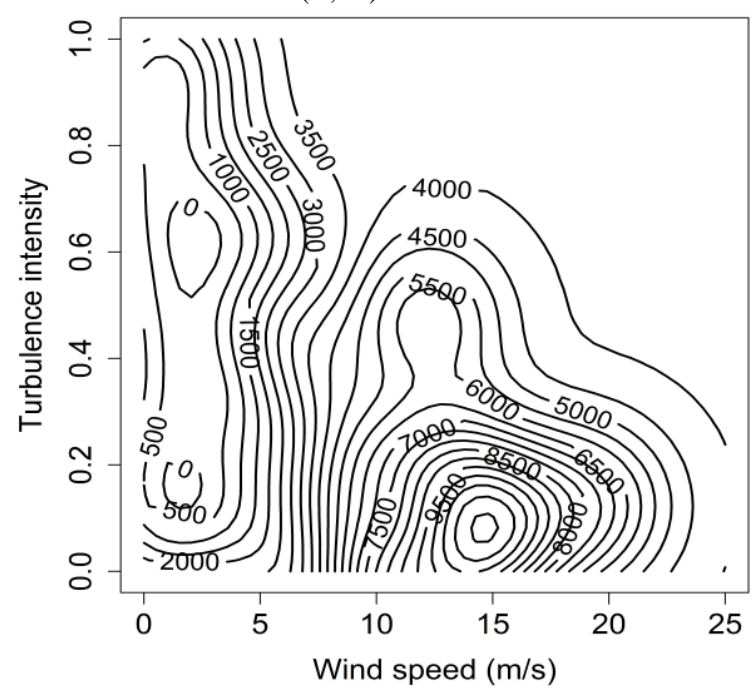

(d) Surrogate with MET01 speed (in-wake)

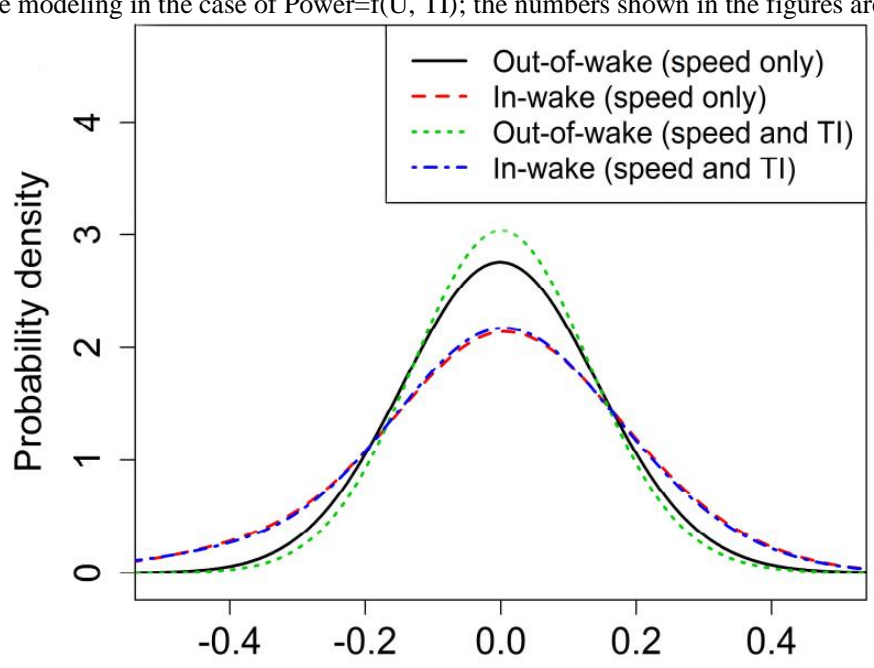

Normalized error by 9 turbines rated power

Figure 13. Uncertainty quantification in the case of nine turbines

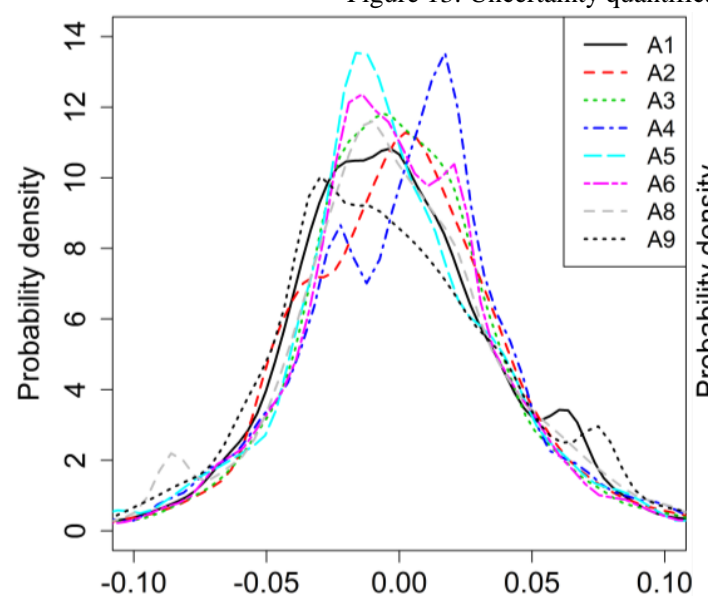

Normalized error by turbine's rated power

(a) W.R.T. turbine's own speed

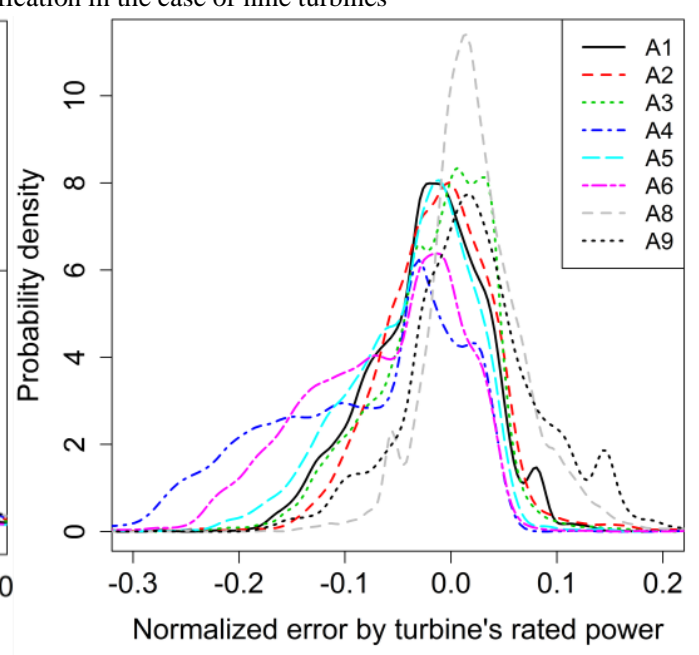

(b) W.R.T. MET tower speed 


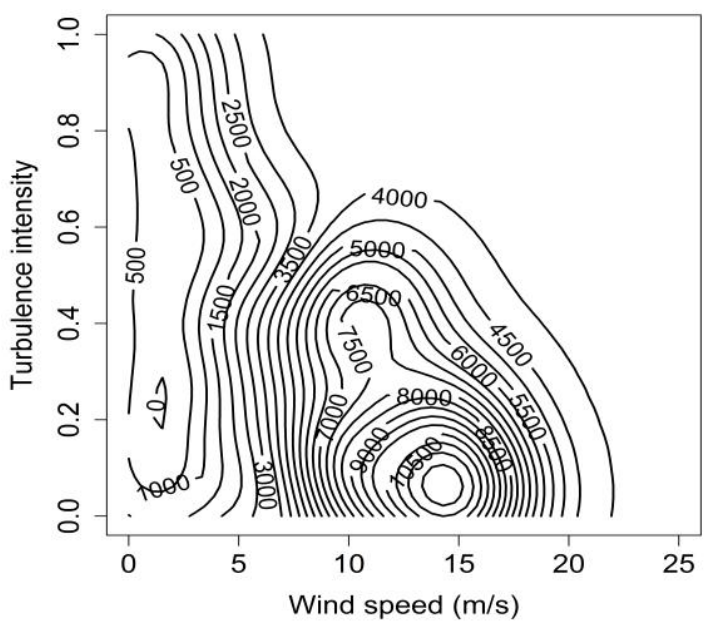

(a) Contour plot of surrogate model Figure 15. SVM using turbine's own wind speed and turbulence intensity for summer months
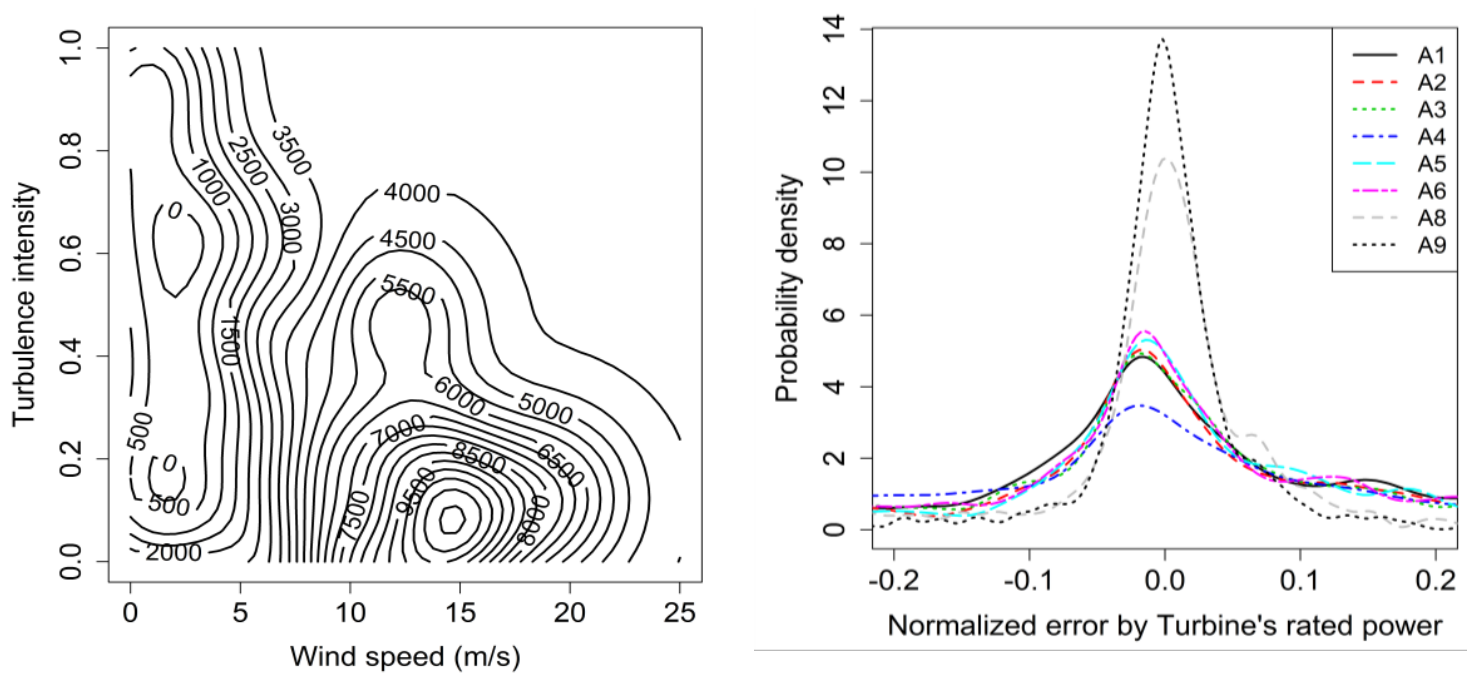

(a) Contour plot of surrogate model

(b) Uncertainty quantification Figure 16. SVM using MET tower wind speed and turbulence intensity for summer months

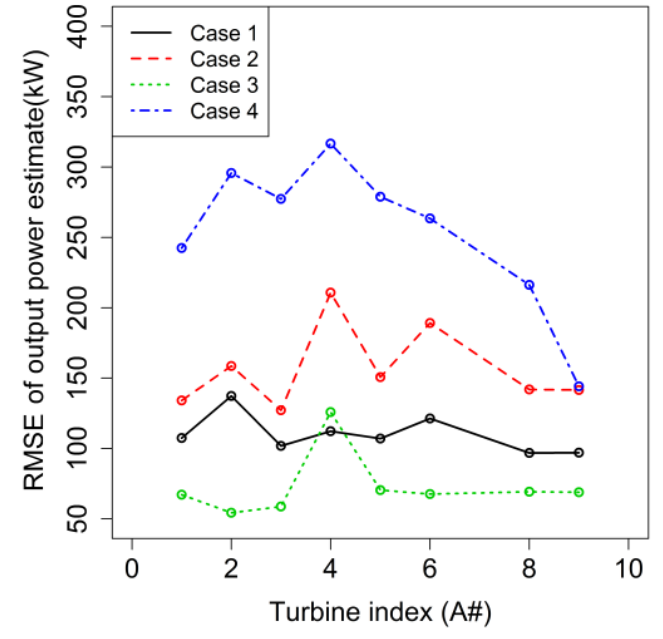

(a) RMSE

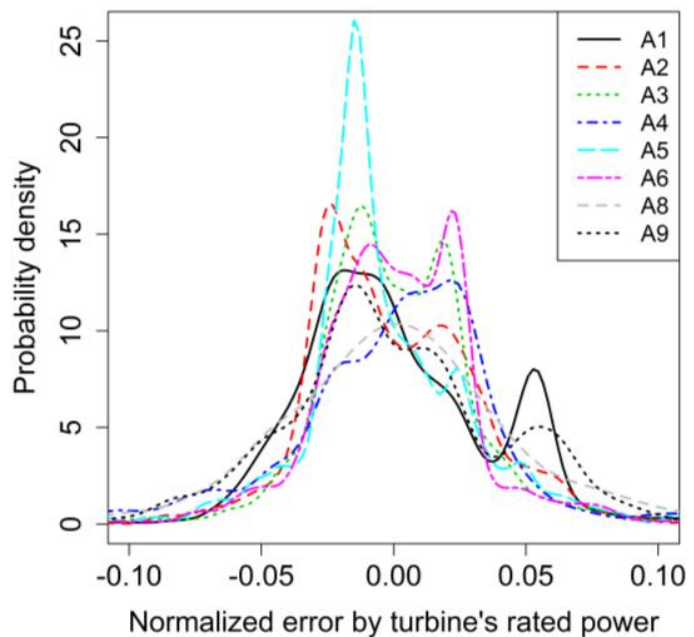

(b) Uncertainty quantification 


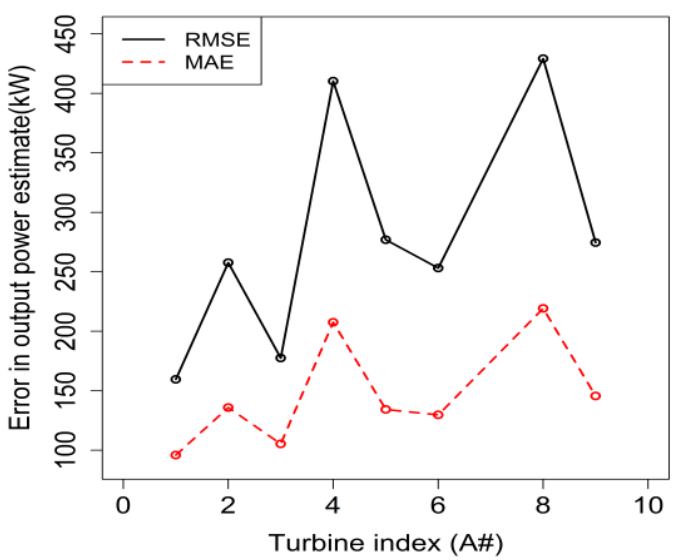

